\title{
Testing Time for Toxicologists
}

by our Special Correspondent

A CHALlenge has been thrown out to toxicologists and cancer researchers by the growing demands that new and existing drugs should be tested for carcinogenicity. At present the resources of materials and techniques available to them are limited and they must quickly look for new and improved methods. Taking up the challenge, the Drug Research Board of the US National Academy of Sciences called a conference in Washington DC on May 23-25 to consider carcinogenesis testing in the development of new drugs.

The essence of the procedures most commonly used to test for carcinogenesis has been to treat animalsusually rats and mice-with the drug for varying times, to look for effects on the time of appearance and the number and types of tumours that develop. There are, however, many problems. For example, what strain of animal should be used; how much drug should it receive for how long; and what diet should it eat while being dosed? These, and all the other questions tackled at the meeting, were first thrashed out during day-long discussion groups, whose leaders reported their recommendations during the second and third days.

Dr M. B. Shimkin (University of California, San Diego) recommended, on behalf of his group, that inbred strains of mice provide the best single animal for testing, but that rats are also desirable for this purpose, with the Syrian hamster probably the next best species. Larger animals, including primates, however, are impractical for bioassays and should be restricted to special problems. Nevertheless, the use of more non-rodent species should be explored.

The group concerned with the size of doses recommended, through its leader Dr L. Friedman (Food and Drug Administration), the use of the maximum dose that permits valid interpretation of the experimental observations. Usually, the group decided, with rodents this dose should be such that it will not depress the increase in weight more than $10 \%$ compared with controls. This dose should also leave $50 \%$ of treated animals alive at the end of the study and produce no pharmacological effects to invalidate the results. Two other doses should be used, however, the second highest usually being 10-50\% of the maximum. Dr Friedman's group recommended that treatment, which should begin at conception and continue until completion of the study, or death, should be given on five to seven days each week. The route of administration to the animals should be oral or topical when they apply to human use of the drug. Intramuscular and subcutaneous injection should be used only if absorption is poor in the test animal after oral administration, and inhalation should be limited because animal models are not sufficiently established to yield valid negative results with substances of unknown carcinogenic potency.

Diet can affect considerably the progress of carcinogenesis in rodents, and so the choice of food for test animals is crucial, as Dr P. M. Newberne (Massachusetts Institute of Technology) reported. His group recommended that diets for carcinogenesis testing should be standardized, so that results from different laboratories can be compared and reproduced. For this purpose they would like to see manufacturers producing semi-synthetic diets free of known enzyme inhibitors and antioxidants, and containing standardized amounts of protein, carbohydrates, vitamins and minerals.

Some of the problems besetting the hard-pressed pathologist who has to interpret the results of these various carcinogenesis tests were described by Dr W. Richter (University of Chicago), giving one of the individual contributions to the conference. Sometimes a supposed tumour turns out to be an abscess, or there are cases such as the hyperplastic rodent bladder which looks, in the microscope, like a normal primate bladder.

Considerable energy is now being devoted to the development of tests which can be carried out more quickly than those involving live animals. Dr J. A. DiPaulo (National Institutes of Health) said that some tests using a Syrian hamster cell culture system at the National Cancer Institute can be completed in ten days. Speaking for the discussion group concerned with tissue culture techniques, Dr B. Weinstein (Columbia University, New York) said that modesty is required in anticipating the outcome of tests of carcinogenicity on, as well as hamster cells, mouse fibro. blasts and epithelial cells, which come principally from the rat liver. The problems of using tissue of human origin also seem to be legion, as the discussion group charged with considering this question discovered. Dr A. Freeman (Children's Hospital, Akron, Ohio) reported the recommendations that organ culture methods must be developed for testing carcinogens, and that cell banks should be established. His group also suggested that a committee should be set up to help carcinogenesis testers to obtain human tissue from inbred populations, which may be predisposed to cancer, in remote geographical areas. When it comes to obtaining the necessary evidence from the transplantation of transformed cells into donors (to establish whether they develop cancer), there are obvious objections to the use of human subjects. Dr Freeman's group therefore recom. mended that evidence should be accepted from xenografts, but only with concomitant and careful pathological investigation.

The general feeling of the conference, summarized by Dr D. T. Rall (National Institute of Health Sciences), was that the life-time studies of whole animals are essential to carcinogenesis testing, but that short term methods also have their function.

\section{MEMBRANE TRANSPORT \\ Transduction Deductions}

from our Molecular Biology Correspondent

THE study of active transport is a thriving industry at the heart of which lies a kernel of profound ignorance: it is simply not known how the hydrolysis of ATP occasions the transfer of ions through the membrane against a concentration gradient. Many facts about the process have been amassed, one of the most salient of which emerged only recently, namely that the calcium ATPase of sarcoplasmic reticulum at least can be made to go into reverse, to function, so to speak, as a motor instead of a dynamo, and synthesize ATP from ADP and phosphate, when the concentration gradient is allowed to run down. A facet which may be common to all ATPase systems is the ability to catalyse the exchange of oxygen between water and phosphate, which, it is supposed, is in some way related to a step in the ATPase mechanism. Boyer and his associates have shed some light on this dark corner, and two new articles on ATPases of quite different kinds again point to an underlying unity.

Dahms and Boyer (J. biol. Chem., 248, 3155 ; 1973) have examined the sodium-potassium ATPase from kidney membrane and from the electroplax of the electric eel. The preparations of these enzymes catalyse the rapid entry of ${ }^{18} \mathrm{O}$ from isotopically enriched water into phosphate ions in the medium. This is clearly related to the primary enzymatic function, for it requires potassium and is annihilated by sodium, and also ouabain, which is a specific inhibitor of sodium-potassium ATPase. It also requires the presence of magnesium. It is unaffected, on the other hand, by oxidative phosphorylation uncoupling agents, such as dinitrophenol, and it differs also from the mitochondrial system in requiring no nucleotide. Moreover there is no exchange between ATP and either water or orthophosphate. 